\title{
AVALIAÇÃO DA ADESÃO AO CHECKLIST CIRÚRGICO ${ }^{1}$
}

\author{
EVALUATION OF COMPLIANCE WITH THE SURGICAL CHECKLIST
}

\section{EVALUACIÓN DE LA ADHESIÓN A LA LISTA DE VERIFICACIÓN QUIRÚRGICA}

\author{
Camila Sarmento Gama* \\ DÉbora Fernanda Silva** \\ Adriana Cristina De Oliveira***
}

\begin{abstract}
RESUMO
Objetivo: Verificar a adesão ao checklist cirúrgico obtida por meio de diferentes ferramentas: completude, auditoria de processo e a avaliação da taxa de complicaçóes e mortalidade, em um hospital universitário. Material e Método: Tratou-se de um estudo transversal realizado em um centro cirúrgico de um hospital universitário de Belo Horizonte, Brasil. A coleta de dados se deu em dois momentos: primeiro, auditoria da aplicaçáo verbal do checklist e comparação com o preenchimento do formulário; e o segundo, de forma paralela, por revisão de prontuários de pacientes de cirurgias colorretais para avaliação da completude, taxas de infecção do sítio cirúrgico, reoperação, reinternação e óbito. Foi realizada análise descritiva dos dados, estatística inferencial e teste qui-quadrado. Resultados: No primeiro momento, 100 procedimentos aleatórios foram acompanhados para auditoria da aplicaçáo verbal e nenhum deles foi totalmente seguido pela equipe cirúrgica, porém os formulários de $65 \%$ foram completamente preenchidos. No segundo momento, dos 353 prontuários analisados, 63,7\% dos checklists estavam completos, a taxa de infecção do sítio cirúrgico foi maior naqueles incompletos e não foram observados impactos da sua completude nos demais desfechos. Conclusão: A completude do checklist não retratou a adesão ao mesmo, devendo ser usada com cautela como indicador, associando-a ferramentas complementares como a auditoria periódica e o monitoramento dos desfechos clínicos.
\end{abstract}

Palavras-chave: Segurança do Paciente; Cirurgia; Lista de Checagem; Centros Cirúrgicos; Indicadores de Qualidade em Assistência à Saúde.

${ }^{1}$ Apoio Financeiro: Coordenação de Aperfeiçoamento de Pessoal de Nível Superior - CAPES; Fundação de Amparo à Pesquisa de Minas Gerais - FAPEMIG; Conselho Nacional de Desenvolvimento Científico e Tecnológico.

*Doutora. Prefeitura de Belo Horizonte. Secretaria Municipal de Saúde, Belo Horizonte, Minas Gerais, Brasil. ORCID: https:// orcid.org/0000-0002-9126-7522 Email: camilasarmentogama@gmail.com. Autor correspondente.

**Enfermera. Escola de Enfermagem. Universidade Federal de Minas Gerais, Belo Horizonte, Minas Gerais, Brasil. ORCID: https://orcid.org/0000-0002-8076-1197 Email: debora.fernanda8silva@gmail.com.

***Doutora. Departamento de Enfermagem Básica. Escola de Enfermagem. Universidade Federal de Minas Gerais, Belo Horizonte, Minas Gerais, Brasil. ORCID: https://orcid.org/0000-0002-4821-6068 Email: adrianacoliveira@gmail.com. 


\section{ABSTRACT}

Objective: To verify surgical checklist compliance obtained through different tools: checklist completion, process auditing and evaluation of complication and mortality rates in a university hospital. Materials and Methods: Cross-sectional study carried out in a surgical center of a university hospital in Belo Horizonte, Brazil. Data collection occurred in two stages: firstly, by auditing the oral application of the checklist and comparing it with the checklist completion level; and secondly, by reviewing medical records of colorectal surgery patients to evaluate compliance, surgical site infection, reoperation, readmission and mortality rates. Descriptive data analysis, inferential statistics and chi-square test were performed. Results: In the first stage, 100 random procedures were monitored for oral application auditing and none of them were totally applied by the surgical team, however, $65 \%$ of the checklists were fully completed. In the second stage, from 353 analyzed records, $63.7 \%$ of the checklists were complete, surgical site infection rate was higher in cases with incomplete checklists and no impact of their compliance level on the other outcomes was observed. Conclusion: Checklist completion does not portray its compliance level and should be used with caution as an indicator, associating it with complementary tools such as regular auditing and monitoring of clinical outcomes.

Key words: Patient Safety; Surgery; Checklist; Surgical Centers; Quality Indicators in Health Care.

\section{RESUMEN}

Objetivo: Verificar el cumplimiento de la lista de verificación quirúrgica obtenida por diferentes herramientas en un hospital universitario: completación, auditoría de procesos y evaluación de la tasa de complicaciones y mortalidad. Material y Método: Se realizó un estudio transversal en un centro quirúrgico en un hospital universitario en Belo Horizonte, Brasil. La recolección de datos se realizó en dos momentos: primero, la auditoría de la aplicación oral de la lista de verificación y la comparación con el formulario de la lista de verificación; y segundo, en paralelo, mediante la revisión de los registros médicos de pacientes sometidos a cirugías colorrectales para la evaluación del cumplimiento, tasas de infección del sitio quirúrgico, reoperación, readmisión y mortalidad. Se realizó un análisis descriptivo de los datos, estadística inferencial y prueba de chicuadrado. Resultados: En el primer momento se siguieron 100 procedimientos aleatorios para la auditoría de la aplicación oral de la lista y ninguno de ellos fue totalmente aplicado por el equipo quirúrgico, sin embargo, $65 \%$ de los formularios estaban completamente llenos. En el segundo momento, de los 353 registros analizados, $63,7 \%$ de las listas de verificación estaban completas, la tasa de infección del sitio quirúrgico fue mayor en las listas incompletas y no hubo impactos de su cumplimiento en los otros resultados. Conclusión: La completación de la lista de verificación no fue un retrato de su adhesión y debe usarse con precaución como un indicador, asociándolo con herramientas complementarias, como la auditoría periódica y el seguimiento de los resultados clínicos.

Palabras clave: Seguridad del Paciente; Cirugía; Lista de Verificación; Centros Quirúrgicos; Indicadores de Calidad de la Atención de Salud.

Fecha de recepção: 02/06/2020

Fecha de aceitação: 26/09/2020

\section{INTRODUÇÁO}

As complicações cirúrgicas acometem entre 3 e 16\% dos pacientes cirúrgicos e a mortalidade entre 0,4 e $0,8 \%$, podendo chegar entre 5 e $10 \%$ em países em desenvolvimento, sendo metade considerada evitável $^{(1)}$. Com o objetivo de minimizar essas complicações evitáveis, em 2008, a Organização Mundial da Saúde (OMS) propôs o Segundo Desafio Global para a Segurança do Paciente com a campanha "Cirurgias Seguras Salvam Vidas", incentivando a adoção de um checklist com o propósito de despertar as equipes cirúrgicas para etapas simples, porém críticas do procedimento 
operatório e favorecer a comunicação entre os profissionais envolvidos nesse cuidado ${ }^{(1)}$.

Em 2009, a OMS realizou um estudo piloto implementando o checklist cirúrgico em diferentes realidades socioeconômicas e constatou uma redução significativa nas taxas de complicaçóes cirúrgicas: de $11 \%$ para $7 \%$ e de óbito de $1,5 \%$ para $0,8 \%{ }^{(2)}$. Contudo, desde então, a sua implementação não tem evidenciado resultados semelhantes em alguns cenários ${ }^{(3-5)}$, verificando-se que aspectos como aceitaçáo cultural, capacitação deficiente e estratégia de implementação falha ${ }^{(5,6)}$ implicam em baixa adesão ao instrumento pelas equipes de enfermagem, cirúrgica e anestésica e, consequentemente, ausência de impactos significativos para a segurança do paciente cirúrgico ${ }^{(3,7)}$.

No Brasil, em 2013, a Agência Nacional de Vigilância Sanitária recomendou, por meio da Resolução Diretoria Colegiada de número 36, a adesão das instituições de saúde a protocolos básicos da OMS, incluindo o programa "Cirurgias Seguras Salvam Vidas"(8). Ainda que muitos estabelecimentos de saúde tenham implantado o programa, o principal gargalo tem sido a geraçáo de indicadores que favoreçam a proposição de açôes visando a sua melhoria. Assim, pode se afirmar que, no Brasil, a implementação tem sido incipiente, devido ao reduzido número de resultados que propiciem aprimoramento das açóes ou o alcance dos objetivos propostos pelo programa ${ }^{(9-12)}$.

A mensuração da adesão ao uso do checklist por meio de indicadores como a completude do formulário, a auditoria clínica e a verificação dos seus impactos em morbimortalidade, fornecem dados importantes às instituiçóes de saúde acerca da sua implementação e eficácia, contribuindo para o planejamento de açóes voltadas para a assistência ao paciente cirúrgico pelos gestores e profissionais da saúde ${ }^{(1,11)}$.

Contudo, observa-se uma preocupação das instituições em avaliar apenas a completude do checklist cirúrgico obtida por meio de registros dos prontuários como um indicador, sem mensurar os impactos clínicos ou verificar a rotina do seu emprego nas salas operatórias ${ }^{(3,11)}$. Embora seja um indicador utilizado amplamente para mensurar o uso do checklist cirúrgico, a completude tem se mostrado falha para representar a sua adesão ${ }^{(5,11)}$, sendo importante investigar a adesão por outros meios. Assim, objetivou-se verificar a adesão ao checklist cirúrgico, obtida por meio de diferentes ferramentas: completude, auditoria de processo e a avaliação da taxa de complicaçóes e mortalidade, em um hospital universitário.

\section{MATERIAL E MÉTODO}

Realizou-se um estudo quantitativo transversal em um hospital público e universitário de Belo Horizonte, cujo checklist cirúrgico foi implementado em 2014. A coleta de dados foi realizada em dois momentos: o primeiro foi uma auditoria de processo para verificação da aplicação verbal, na sala operatória e completude do checklist cirúrgico na prática clínica pelo circulante de sala; e o segundo, uma análise de prontuário para verificar a relação da completude do checklist e o impacto dessa na infecção do sítio cirúrgico (ISC), reoperação, readmissão e óbitos no seguimento de 30 dias no pós-operatório.

No primeiro momento, na auditoria de processo na sala de cirurgia, a coleta de dados foi conduzida por uma bolsista de iniciação científica, previamente treinada pelo pesquisador principal para o acompanhamento das cirurgias eletivas de diferentes especialidades que atenderam aos seguintes critérios: duração superior à uma hora e uso do checklist cirúrgico. Os procedimentos eletivos acompanhados na instituição foram aqueles realizados de segunda a sexta-feira de abril a novembro de 2018.

A fim de evitar o Efeito Hawthorne, que corresponde à modificação do comportamento dos indivíduos sabidamente observados ${ }^{(13)}$, os profissionais presentes na sala operatória foram informados da coleta de dados ao fim do estudo, sendo então apresentado o termo de consentimento livre e esclarecido (TCLE) a esses profissionais, no sentido de solicitar sua anuência para utilização das observaçóes do procedimento cirúrgico sob sua responsabilidade.

A execução do checklist cirúrgico na instituição do estudo é feita de forma oral com o auxílio de um instrumento elaborado pelo setor em papel impresso que é anexado ao prontuário do paciente em cada cirurgia. A equipe de enfermagem atuando como circulante de sala é responsável pela sua condução e registro, efetuando a verificação dos itens por meio de perguntas direcionadas ao paciente, equipe 
cirúrgica e anestésica.

Um instrumento foi elaborado com base no checklist utilizado na instituiçáo para que a bolsista acompanhasse, em cada cirurgia, o preenchimento do formulário à medida que observava: a confirmação verbal do checklist realizada por algum membro da equipe cirúrgica, a participação coletiva ou não da equipe nos distintos momentos da sua aplicação e o responsável pela sua condução. Ao final da cirurgia, após a saída da equipe da sala operatória, a bolsista checava os itens preenchidos no checklist pela circulante de sala e comparava com os itens checados em voz alta pela equipe nos distintos momentos da cirurgia. Assim, procedia a anotação do registro do checklist pela circulante e o que de fato havia sido conferido verbalmente pela equipe. As etapas do checklist da instituição nas suas três fases, em consonância com a proposta original da OMS, estáo apresentados a seguir: 1) Entrada (Sign in): confirmação pela equipe do nome completo do paciente; conferência da funcionalidade dos equipamentos; demarcaçáo do sítio cirúrgico; reserva de hemoderivados; verificação da via aérea; identificação de alergia; e administração de antibiótico profilático entre 30 e 60 min antes da incisão; 2) Pausa cirúrgica (Time out): apresentação dos membros da equipe cirúrgica pelo nome e função; confirmação verbal da identificação do paciente, sítio cirúrgico e procedimento; planejamento das etapas críticas da cirurgia; confirmação da reserva de hemoderivados; previsão do tempo cirúrgico; identificação da estabilidade hemodinâmica do paciente; disponibilidade e funcionamento dos equipamentos; e conferência da esterilização e validade dos materiais necessários; 3) Saída (Sign out): identificação das peças cirúrgicas com etiqueta para encaminhamento à anatomia patológica e assinatura do responsável pelo preenchimento do checklist.

No segundo momento, foi conduzida uma análise de prontuário para verificar a relação da completude do checklist e o impacto dessa em ISC, reoperação, readmissão e óbitos no seguimento de 30 dias no pós-operatório de cirurgias colorretais, conduzida pelo pesquisador principal e duas bolsistas de iniciação científica, previamente treinadas para a coleta dos dados. Nessa etapa, foram analisados prontuários de pacientes $\geq 16$ anos submetidos a cirurgias da coloproctologia de 1 de janeiro de 2015 a 31 de julho de 2017. Essa especialidade foi selecionada por representar um grande volume cirúrgico na instituição e por ter maiores taxas de ISC, ao realizar predominantemente cirurgias potencialmente contaminadas ou contaminadas.

Nessa fase, avaliou-se registros da completude do checklist e da ocorrência de ISC; retorno ao centro cirúrgico após a alta do mesmo para reoperaçáo decorrente de complicação da cirurgia prévia; reinternação após alta hospitalar relacionada a cirurgia; e mortalidade em decorrência do procedimento, que tenham ocorrido no período de 30 dias após a cirurgia.

Foram excluídos todos os registros de pacientes submetidos a cirurgias da coloproctologia infectadas, de emergência, por via laparoscópica, que estiveram em uso de antimicrobiano terapêutico e que não possuíam checklist.

A completude/adesão ao checklist foi considerada total quando todos os itens que compunham o instrumento da instituição foram preenchidos/ executados, e parcial quando mais de um item ficou sem preenchimento/execução. Para fins de esclarecimento o termo "completude" utilizado no estudo se refere ao preenchimento do checklist e "execução" ou "adesão" à sua checagem oral.

Os dados foram digitados e analisados com o auxílio do software estatístico SPSS (Statistical Package for the Social Sciences) versão 21.0. Foi realizada uma análise descritiva e inferencial dos dados com teste qui-quadrado, considerando-se o valor de $\mathrm{p}<0,05$ estatisticamente significativo.

A coordenação médica e de enfermagem do centro cirúrgico esteve ciente da coleta de dados. O projeto foi aprovado pelo Comitê de ética em Pesquisa da Universidade Federal de Minas Gerais - 037048/2017 (CAAE 30783614.3.0000.5149).

\section{RESULTADOS}

\section{Primeiro momento, auditoria de processo e com-}

pletude: Foram monitorados 100 procedimentos cirúrgicos cuja média de duração foi de 155,6 min (mínimo de 60 e máximo de $532 \mathrm{~min}$ ), sendo considerado para o cálculo o tempo decorrido entre o início e o término do procedimento cirúrgico. As especialidades acompanhadas foram: mastologia (18\%), ginecologia (15\%), cirurgia geral (12\%), aparelho digestivo (11\%), plástica (10\%), pediatria 
$(8 \%)$, ortopedia $(8 \%)$, cabeça e pescoço $(4 \%)$, coloproctologia (4\%), otorrinolaringologia (3\%), cardiovascular $(3 \%)$, urologia $(2 \%)$, neonatologia $(1 \%)$ e torácica $(1 \%)$.

Quanto à auditoria conduzida para sua aplicação verbal, nenhum checklist foi inteiramente executado pela equipe cirúrgica, ao passo que sua completude total no formulário da sala de cirurgia, pelo circulante de sala alcançou $65 \%$ do total. A Figura 1 compara a completude do impresso e a sua real execução na sala cirúrgica nos itens da "entrada" do checklist, tendo sido encontrada maior correspondência entre a adesão e completude ao item "administração da antibioticoprofilaxia".

A Figura 2 compara a completude e a execução dos itens na "pausa cirúrgica" do checklist. Nesse momento a completude correspondeu totalmente à adesão ao item "confirmação da esterilização dos instrumentais”.

Por fim, a Figura 3 compara a completude e a execução dos itens na "saída" do checklist, tendo sido observada uma discrepância entre a sua completude e adesão ao item "identificação das amostras".

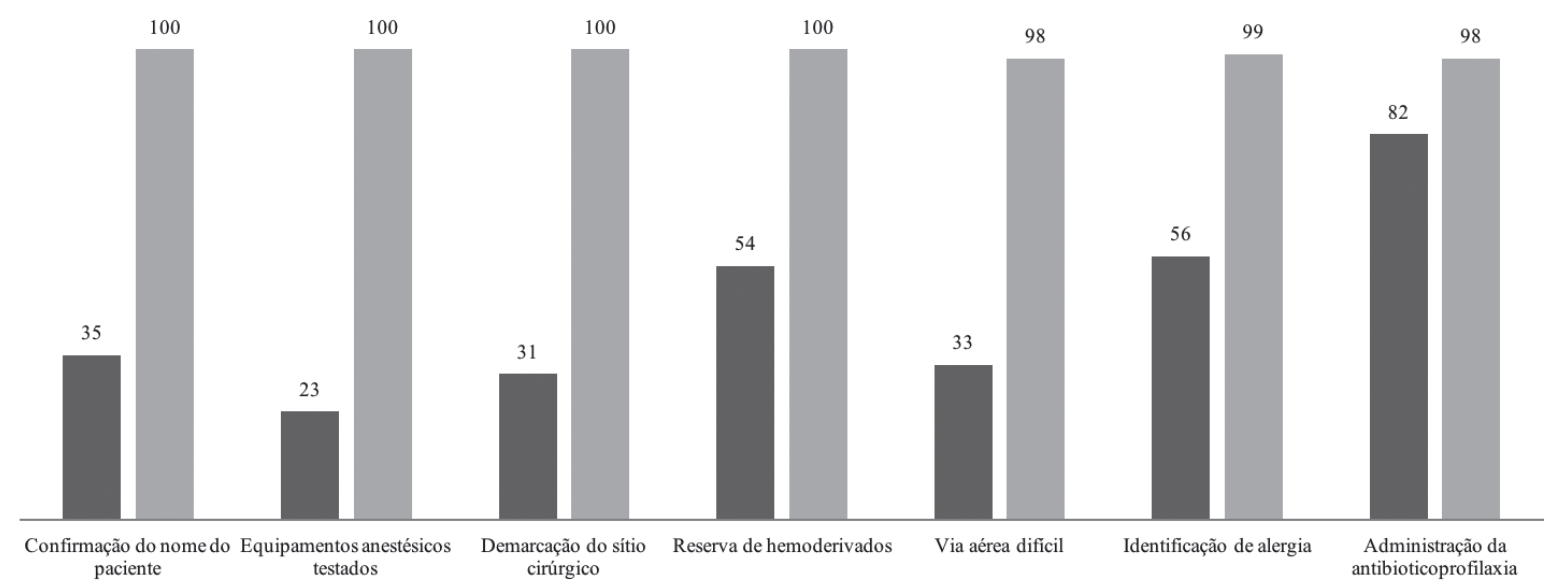

Figura 1. Preenchimento e execução dos itens na "entrada" do checklist cirúrgico. Belo Horizonte, MG, Brasil, 2018.

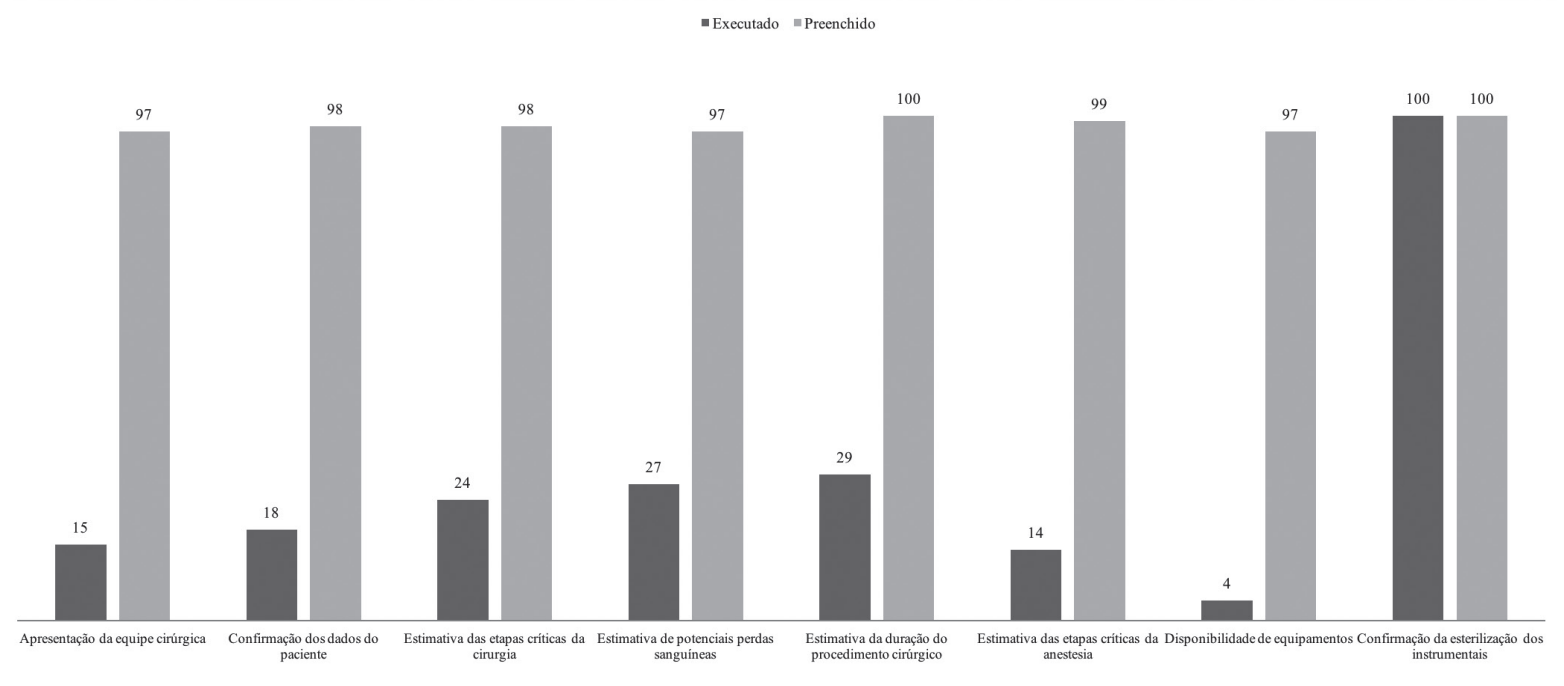

Figura 2. Preenchimento e execução dos itens na "pausa cirúrgica" do checklist cirúrgico. Belo Horizonte, MG, Brasil, 2018. 




Figura 3. Preenchimento e execução dos itens na "saída" do checklist cirúrgico. Belo Horizonte, MG, Brasil, 2018.

Em relação à execução dos momentos do checklist, evidenciou-se que em somente 10 (10\%) dos procedimentos a etapa denominada "entrada" foi totalmente executada, ao passo que a completude do formulário pelo circulante de sala dessa etapa, foi identificada em 95 (95\%) instrumentos. A "pausa cirúrgica" não foi executada completamente em nenhuma cirurgia, mas esse momento estava totalmente preenchido em 90 (90\%) checklists. De maneira semelhante, a "saída" também não, mas foi completamente preenchida em 74 (74\%).

Em todos os procedimentos assistidos, o técnico de enfermagem foi o responsável pelo preenchimento do checklist e pela sua condução verbal em 92 (92\%), sendo as questôes da "entrada" e "saída" direcionadas para a equipe cirúrgica e as da "pausa cirúrgica" para a equipe cirúrgica e anestésica. Destaca-se que em nenhuma cirurgia o checklist foi conduzido verbalmente por completo com a participação de toda a equipe e em oito (8\%) procedimentos não houve responsáveis pela sua condução.

Com relação à participação dos profissionais na "entrada", no item "confirmação do nome do paciente" das 35 confirmaçôes verbais realizadas, cinco $(14,3 \%)$ foram conduzidas pelos cirurgióes isoladamente e $30(85,7 \%)$ pelos técnicos de enfermagem sem a presença do restante da equipe; todas as 23 confirmaçóes orais de conferência dos equipamentos anestésicos foram realizadas por técnicos de enfermagem e anestesistas em conjunto; todas as 31 demarcaçóes do sítio cirúrgico foram realizadas por cirurgióes dentro da sala operatória; as confirmaçóes verbais da reserva de hemoderivados foram executadas pelo técnico de enfermagem e cirurgião em 54 (54\%) procedimentos; a identificação de via aérea difícil foi realizada pelo técnico de enfermagem e anestesista em 33 (33\%) procedimentos; a identificação de alergia foi executada em $56(56 \%)$ procedimentos, sendo que em 14 (25\%) casos o técnico de enfermagem foi o responsável por essa etapa, em $41(73,2 \%)$ casos o anestesista e em um $(1,8 \%)$ o médico cirurgiáo, sempre de forma isolada e não com participação multidisciplinar conforme prevê o protocolo; a confirmação oral da antibioticoprofilaxia foi feita pelo técnico de enfermagem, cirurgiáo e anestesista em conjunto em 82 (82\%) procedimentos.

Em relação à "pausa cirúrgica", observou-se o trabalho em equipe na execução de alguns itens propostos em $32(32 \%)$ procedimentos, com a realizaçáo da pausa de fato e participação da equipe multidisciplinar. $\mathrm{Na}$ "saída", a "identificação de amostras" foi realizada em conjunto por técnicos de enfermagem e cirurgióes nos procedimentos em que foram executadas.

Segundo momento, completude da análise por prontuários e mensuraçáo dos impactos: No período de 1 de janeiro de 2015 a 31 de julho de 2017 foram encontrados e avaliados 800 prontuários de pacientes submetidos a cirurgias da coloproctologia. Desse total, 353 foram incluídos na análise, de acordo com os critérios do estudo, dos quais $225(63,7 \%)$ checklists estavam completos e 128 (36,3\%) incompletos. A média de duração das cirurgias avaliadas foi de 184,2 min (mínimo 
de 60 e máximo de $600 \mathrm{~min}$ ), sendo considerado para o cálculo o tempo decorrido entre o início e o término do procedimento cirúrgico.

Quanto à completude dos checklists verificados no prontuário constatou-se que $48 \quad(37,5 \%)$ estavam incompletos na "entrada", $43(33,6 \%)$ na "pausa cirúrgica", 23 (18\%) simultaneamente na "entrada" e "pausa cirúrgica”, sete (5,5\%) na "saída", três $(2,3 \%)$ na "pausa cirúrgica" e "saída", três $(2,3 \%)$ na "entrada" e "saída" e um $(0,8 \%)$ estava incompleto em todas as três fases. A completude dos itens integrantes do formulário do checklist é apresentada na Figura 4.
E quanto aos desfechos propostos no estudo para serem analisados até o $30^{\circ}$ dia do pós-operatório, nos prontuários avaliados observou-se a ocorrência dos seguintes: óbito em $12(3,4 \%)$ casos, reoperação em $28(7,9 \%)$, reinternação em seis $(1,7 \%)$ e ISC em $50(14,2 \%)$ e a sua relação com a completude do checklist (Figura 5). De acordo com a Figura 5, embora tenha sido observado um aumento do percentual de reoperaçôes, reinternaçóes e ISC em formulários de checklists incompletos, somente o último foi estatisticamente significativo pelo teste qui quadrado.



Figura 4. Completude dos itens do checklist na "Entrada", "Pausa cirúrgica" e "Saída". Belo Horizonte, MG, Brasil, 2018.

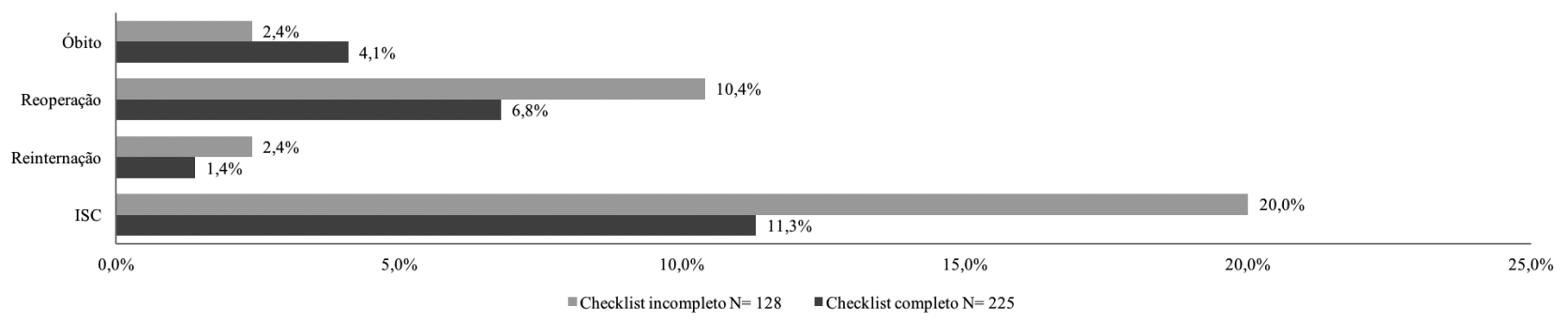

Figura 5. Distribuição dos desfechos por completude do checklist cirúrgico. Belo Horizonte, MG, Brasil, 2018. 


\section{DISCUSSÃO}

Os achados do presente estudo demonstraram que a completude do checklist utilizada como indicador de adesão ao mesmo, não corresponde à sua prática na realidade, o que reflete em baixos impactos para a segurança do paciente, dado corroborado por diversos autores ${ }^{(7,11,14)}$.

A comparação entre dados de registro da completude do checklist, avaliação do seu impacto em desfechos clínicos e auditoria durante a cirurgia evidenciou que a última foi o melhor indicador para retratar a realidade da adesão ao checklist. As auditorias de processos, semelhante à que foi conduzida no estudo, visam garantir a qualidade da assistência prestada pelas instituiçóes de saúde. $\mathrm{O}$ seu sucesso depende da periodicidade, da definição de metas, disponibilização de ferramentas e recursos adequados, bem como comunicação eficaz. Além de identificar a adesão ao processo, ela auxilia na compreensão das barreiras à sua implementação e, consequentemente, no planejamento de melhorias e adaptaçōes ${ }^{(11,14,15)}$.

Por outro lado, a completude dos formulários do checklist, se mostrou como um indicador frágil na representação da prática, pois a execução dos itens correspondentes foi aquém do registrado com comunicação multidisciplinar deficiente. De maneira semelhante, alguns trabalhos nacionais também identificaram a não correspondência entre a completude do checklist e a sua execução, tendo encontrado na "pausa cirúrgica" preenchimento completo entre 90 e $100 \%$ e, em contrapartida, nenhuma confirmação verbal nos procedimentos avaliados $^{(11,14)}$. Da mesma forma, no cenário internacional, um estudo em cinco hospitais ingleses observou a verificação em formulário de dois terços dos itens do checklist, porém a ausência dos membros da equipe em mais de $40 \%$ dos casos e inexistência da "pausa" em mais de 70\%, tendo a "saída" sido concluída em 39\% dos procedimentos $^{(7)}$.

Com o intuito de aumentar a confiabilidade do indicador de processo gerado pela avaliação da completude dos formulários do checklist, sugerese o uso concomitante da auditoria ou da análise dos desfechos. Segundo a OMS, a vigilância dos procedimentos deve ser conduzida, pois em si, constitui uma forma de avaliar a assistência à saúde, considerando-se que o processo influencia diretamente no resultado ${ }^{(1)}$, podendo mitigar/ reduzir os eventos adversos. No presente estudo, a auditoria, como mecanismo de vigilância e proposiçâo de um indicador da realidade da aplicação e adoção pelas equipes do checklist cirúrgico, evidenciou falhas ainda existentes no processo de implementação (completude/adesão) do checklist, apesar de ter sido adotado no serviço desde 2014. A inexistência de estratégias de implementação do checklist fundamentada em análise prévia do ambiente, cultura institucional, envolvimento multiprofissional e treinamento de toda a equipe, a fim de favorecer seu envolvimento, podem resultar em comunicação frágil, resistência ao processo, despreparo e desinteresse na sua adoção pela falta de percepção de seu real impacto na segurança do paciente, do procedimento, da instituição e da equipe ${ }^{(6,12,16)}$.

A análise dos desfechos clínicos também gera indicadores que podem ser utilizados de forma complementar à avaliação da completude do checklist, sendo denominados indicadores de resultado. Comparado ao indicador de processo resultante da auditoria, o indicador de resultado, possui menor ônus já que demanda menos recursos humanos e tempo, uma vez que utiliza dados monitorados constantemente pelas instituiçóes de saúde para verificação do sucesso ou não da implementação de alguma estratégia/política, como taxas de ISC e óbitos $^{(12)}$. Os indicadores de resultado e de processo contribuem para melhor compreensão da realidade na sala operatória, pois favorecem a identificação de lacunas a serem superadas e, consequentemente, o redirecionamento de estratégias na prática clínica ${ }^{(1,}$ 7, 12).

A incoerência entre a completude e a adesão/ impactos encontrados pode ser explicada pela preocupação das instituições em avaliar somente os formulários como indicador de adesão ao processo, sem o seu monitoramento/auditoria na sala operatória, e pelo aumento da pressão para publicar na mídia as taxas de adesão, sem a existência de critérios sobre a forma de coleta dessas informaçôes ${ }^{(3)}$. A divulgaçáo de taxas verificadas por meio de instrumentos preenchidos indiscriminadamente que superestimam a realidade, muitas vezes, atua apenas como ferramentas de propaganda e não como estratégia para a reduçáo de risco de eventos $\operatorname{adversos}^{(3,7)}$.

No Brasil, a maioria dos estudos tem avaliado 
a adesão ao checklist considerando a completude do instrumento, sendo reduzido o número de estudos que avaliaram a sua efetividade em desfechos clínicos e sua execução na prática operatória $^{(11,12,17)}$, demonstrando a necessidade de maior investimento em avaliação da adesão ao checklist obtida complementarmente por outros métodos no território nacional, a fim de aumentar a fidedignidade dos dados coletados e fomentar o planejamento de estratégias que visem à efetividade do processo proposto com a adoção do checklist cirúrgico.

Destaca-se no presente trabalho a baixa adesão/ execução dos itens: "confirmação dos dados do paciente" e "demarcação do sítio cirúrgico". A conferência desses itens é fundamental para evitar a ocorrência de never events, que são eventos totalmente evitáveis e que, por sua vez, nunca poderiam ocorrer como a realização de cirurgias em pacientes equivocados, procedimentos errados ou ainda em regióes anatômicas incorretas e o esquecimento de materiais cirúrgicos no organismo do paciente ${ }^{(18)}$. Os never events são eventos adversos cirúrgicos de grande relevância e impacto, uma vez que geralmente sáo decorrentes de falhas graves que podem provocar incapacidades permanentes ou temporárias ${ }^{(19)}$.

O trabalho em equipe almejado pela OMS ao propor o checklist $^{(1)}$, não foi evidenciado no estudo, e sua premissa é de minimizar as falhas de comunicação que são considerados como causa-raiz de muitos eventos adversos ${ }^{(20)}$, além de promover o trabalho em equipe, quando executado de forma efetiva, o checklist pode contribuir para a melhora do comportamento da equipe cirúrgica e promoçáo e fortalecimento da cultura de segurança no centro cirúrgico $^{(20)}$.

Por outro lado, observou-se que os itens diretamente relacionados à prevenção da $I_{S C}^{(1)}$ como "administração da antibioticoprofilaxia", único conferido em conjunto entre os membros das equipes multidisciplinares na maioria dos procedimentos monitorados, e "confirmação da esterilização dos instrumentais", foram os mais executados durante a auditoria, repercutindo significativamente na redução do percentual de ISC em checklists completos comparados aos incompletos, podendo-se inferir a eficácia do checklist quando usado adequadamente.

$\mathrm{O}$ item "confirmação da esterilização dos materiais" também chama a atenção, pelo fato de ter sido o único aderido e de fato executado. Possivelmente, por ser uma atividade executada pela equipe de enfermagem e, no caso da instituição do estudo, ser conduzida por técnicos de enfermagem que possuem a função de circulantes de sala e coordenadores do checklist, permite-se inferir que as tarefas que essa categoria profissional considera como de sua competência têm sido executadas, ao passo que as demais onde há necessidade de interação profissional com outras categorias, vistas como hierarquicamente superior a sua, têm demonstrado adesão insuficiente.

Ao enfermeiro e equipe de enfermagem compete a segurança do paciente e uma assistência de qualidade. O checklist cirúrgico é uma ferramenta que visa a segurança do paciente cirúrgico e a sua efetividade depende da cultura de segurança organizacional, da compreensão do seu objetivo e relevância para a segurança do cuidado prestado, adaptaçôes à realidade local, liderança, capacitaçôes, monitoramentos e avaliação constantes ${ }^{(1)}$ onde o enfermeiro e a equipe de enfermagem possuem papéis fundamentais como gestor, auditor, pesquisador, professor, coordenador e/ou condutor do processo de implementação e eficácia dessa ferramenta.

Embora o estudo tenha sido conduzido em apenas uma instituição hospitalar, apresenta resultados que possivelmente possam ser expandidos, representando a realidade de muitas instituições de saúde do país e de países em desenvolvimento que têm se adaptado a implementação do checklist cirúrgico.

Destaca-se como limitação do estudo, a inclusão somente de procedimentos da coloproctologia, na análise dos desfechos, porém, justificada pelo alto volume realizado na instituição do estudo. Essa seleção se fez importante por se tratar de uma especialidade com maior volume e diversidade de procedimentos cirúrgicos potencialmente contaminados ou contaminados, cujos pacientes são sujeitos a maiores números de ISC e complicaçôes que podem ser minimizadas com a aplicação do checklist. Contudo, a ausência de intervençóes voltadas para o uso do checklist na instituição em ambos os momentos, permite inferir que não houve mudanças no processo de implementação e, consequentemente, nos desfechos entre o primeiro e o segundo momento avaliados, já que as taxas 
de completude foram semelhantes em ambos os períodos.

\section{CONCLUSÁO}

Os indicadores são ferramentas gerenciais fundamentais para realização de diagnóstico situacional, planejamento de açóes e tomada de decisões devendo, portanto, ser fidedignos à realidade que representam. No presente estudo, evidenciou-se que a completude do checklist utilizada como indicador de adesão ao mesmo, não corresponde à sua prática, refletindo em baixos impactos para a segurança do paciente. A auditoria e o monitoramento do impacto do uso do checklist em desfechos clínicos se mostraram como indicadores de avaliação mais fidedignos à realidade do processo de implementação do checklist.

Assim, conclui-se que a completude do checklist, frequentemente utilizada pelas instituiçôes de saúde como indicador de adesão ao mesmo, deva

\section{REFERÊNCIAS}

1. Organização Mundial da Saúde (OMS). Segundo desafio global para a segurança do paciente: cirurgias seguras salvam vidas [Internet]. Genebra; 2009 [citado 2018 jul 20]. Disponível em: http:// bvsms.saude.gov.br/bvs/publicacoes/seguranca paciente_cirurgias_seguras_salvam_vidas.pdf.

2. Haynes AB, Weiser TG, Berry WR, Lipsitz SR, Abdel-Hadi SB, Dellinger P, et al. A surgical safety checklist to reduce morbidity and mortality in a global population. N Engl J Med [Internet]. 2009 [citado 2018 ago 20]; 360(5): 491-9. Disponível em: http://dx.doi.org/10.1056/NEJMsa0810119.

3. Urbach DR, Govindarajan A, Saskin R, Wilton AS, Baxter NN. Introduction of Surgical Safety Checklists in Ontario, Canada. N Engl J Med [Internet]. 2014 [citado 2018 ago 21]; 370(11): 1029-38. Disponível em: http://dx.doi. org/10.1056/NEJMsa1308261.

4. Zingiryan A, Paruch JL, Osler TM, Hyman NH. Implementation of the surgical safety checklist at a tertiary academic center: Impact on safety culture and patient outcomes. Am J Surg [Internet]. 2017 ser usada com cautela já que pode superestimar a sua aplicação na prática, mascarando a realidade. Sugere-se o seu uso em conjunto com a auditoria ou a análise dos desfechos clínicos como forma de melhorar a sua confiabilidade e garantir a sua eficácia.

Espera-se que o resultado forneça subsídios para a reflexão dos gestores e profissionais de saúde quanto à implementação do checklist em suas instituições, uma vez que apenas a sua implantação no processo de trabalho não assegura a qualidade das práticas e, consequentemente, nem a melhoria na segurança do paciente, sendo necessário investimento da instituição em cultura de segurança, capacitaçôes, estratégias de implementação, adaptaçôes locais, monitoramento da adesão por meio de auditorias e avaliação dos desfechos clínicos. Além disso, pontua-se a necessidade de estudos que avaliem o efeito do uso desses indicadores em longo prazo na eficácia do checklist cirúrgico visando à melhoria da qualidade assistencial e segurança do paciente cirúrgico.

[citado 2018 ago 15]; 214(2): 193-7. Disponível em: http://dx.doi.org/10.1016/j.amjsurg.2016.10.027.

5. Mahmood T, Mylopoulos M, Bagli D, Damignani R, Haji FA. A mixed methods study of challenges in the implementation and use of the surgical safety checklist. Surgery 2019; 165(4): 832-7. doi: https://doi.org/10.1016/j.surg.2018.09.012

6. Kasatpibal N, Sirakamon S, Punjasawadwong Y, Chitreecheur J, Chotirosniramit N, Pakvipas P, et al. An exploration of surgical team perceptions toward implementation of surgical safety checklists in a non-native English-speaking country. Am J Infect Control 2018; 46(8): 899-905. doi: 10.1016/j.ajic.2017.12.003.

7. Russ S, Rout S, Caris J, Mansell J, Davies R, Mayer E, et al. Measuring variation in use of the WHO surgical safety checklist in the operating room: a multicenter prospective cross-sectional study. J Am Coll Surg [Internet]. 2015 [citado 2018 ago 11]; 220(1): 1-11. Disponível em: http://dx.doi. org/10.1016/j.jamcollsurg.2014.09.021.

8. Ministério da Saúde (BR). Agência Nacional de Vigilância Sanitária. Resolução da Diretoria Colegiada 36 de 25 de julho de 2013. D.O.U. Institui açóes para a segurança do paciente em serviços de 
saúde e dá outras providências [Internet]. Brasília: Ministério da Saúde. 2013 [citado 2018 jun 11]. Disponível em: http://portal.anvisa.gov.br/ documents/10181/2871504/RDC_36_2013_ COMP.pdf/36d809a4-e5ed-4835-a3753b3e93d74d5e.

9. Magnago TSBS, Lanes TC, Gonçalves NGC, Ongaro JD, Almeida FO, Dalmolin GL. Avaliação da adesão ao checklist de cirurgia segura em um hospital universitário. Rev Enferm UFSM [Internet]. 2019 [citado 2020 abr 7]; 9(63): 1-15. Disponível em: https://periodicos.ufsm.br/reufsm/ article/view/36780/html.

10. Santana HT, De Freitas MR, Ferraz EM, Evangelista MS. WHO Safety Surgical Checklist implementation evaluation in public hospitals in the Brazilian Federal District. J Infect Public Health [Internet]. 2016 [citado 2018 set 10]; 9(5): 58699. Disponível em: http://dx.doi.org/10.1016/j. jiph.2015.12.019.

11. Oliveira AC, Abreu AR, Almeida SS. Implementação do checklist de cirurgia segura em um hospital universitário. Revista Cofen [Internet]. 2017 [citado 2018 ago 11]; 8(4): 7-12. Disponível em: https://doi.org/10.21675/2357-707X.2017. v8.n4.972.

12. Prates CG, Stadñik CM, Bagatini A, Caregnato RC, De Moura GM. Comparison of surgical infection rates after implementation of a safety checklist. Acta Paul Enferm [Internet]. 2018 [citado 2018 ago 23]; 31(2): 116-22. Disponível em: http:// dx.doi.org/10.1590/1982-0194201800018.

13. Cavadas LF, Ponte C, Granja M. Estudantes de medicina como colaboradores na investigaçáo em centros de saúde. Rev Port Med Geral Fam [Internet]. 2018 [citado 2020 abr 15]; 34(6): 42836. Disponível em: http://dx.doi.org/10.32385/ rpmgf.v34i6.12335.

14. Maziero ECS, Silva AEBC, Mantovani MF, Cruz EDA. Adherence to the use of the surgical checklist for patient safety. Rev Gaúcha Enferm [Internet].
2015 [citado 2018 ago 11]; 36 (4): 14-20. Disponível em: http://dx.doi.org/10.1590/19831447.2015.04.53716.

15. Ray-Barruel G, Ullman AJ, Rickard CM, Cooke M. Clinical audits to improve critical care: Part 2: analyse, benchmark and feedback. Aust Crit Care [Internet]. 2018 [citado 2018 set 18]; 31(2): 106-9. Disponível em: https://doi.org/10.1016/j. aucc.2017.04.002.

16. Molina G, Jiang W, Edmondson L, Gibbons L, Huang LC, Kiang MV, et al. Implementation of the surgical safety checklist in South Carolina hospitals is associated with improvement in perceived perioperative safety. J Am Coll Surg [Internet]. 2016 [citado 2018 jul 11]; 222(5): 72536. Disponível em: http://dx.doi.org/10.1016/j. jamcollsurg.2015.12.052.

17. Gama CS, Backman C, Oliveira AC. Effect of surgical safety checklist on colorectal surgical site infection rates in 2 countries: Brazil and Canada. Am J Infect Control [Internet]. 2019 [citado 2020 abr 15]; 47(9): 1112-7. Disponível em: http:// dx.doi.org/10.1016/j.ajic.2019.03.002.

18. National Health Service England (NHS). Never events lists 2018 [Internet]. 2018 [citado 2018 ago 15]. Disponível em: https://improvement.nhs. uk/documents/2266/Never_Events_list_2018_ FINAL_v5.pdf

19. Tchangai B, Tchaou M, Iroukora Kassegne, Simlawo K. Incidence, root cause, and outcomes of unintentionally retained intraabdominal surgical sponges: a retrospective case series from two hospitals in Togo. Patient Saf Surg [Internet]. 2017 [citado 2018 set 19]; 11(25): 1-7. Disponível em: http://dx.doi.org/10.1186/s13037-017-0140-2.

20. Cabral RA, Eggenberger T, Keller K, Gallison BS, Newman D. Use of a surgical safety checklist to improve team communication. AORN J [Internet]. 2016 [citado 2020 abr 15]; 104(3): 206-16. Disponível em: http://dx.doi.org/10.1016/j.aorn. 2016.06.019. 